\title{
A CONFORMALLY INVARIANT OSEEN EQUATION FOR FLOW AT SMALL REYNOLDS NUMBERS
}

\author{
BY
}

K. B. RANGER

University of Toronto, Toronto, Ontario, Canada

\begin{abstract}
Starting from a complex variable formulation of the exact Navier-Stokes equations for the steady two-dimensional motion of an incompressible viscous fluid, a Burgers type linearization is introduced at small Reynolds number which results in a conformally invariant Oseen equation. The independent variables are the velocity potential and stream function for the corresponding irrotational flow. There are certain analytical advantages in this approach for the determination of uniformly valid approximations to flow at small Reynolds numbers and the method is illustrated by various examples.
\end{abstract}

Introduction and the Oseen approximation. For two-dimensional flow the fluid velocity can be prescribed in terms of a stream function $\Psi$ by

$$
\mathbf{q}=\operatorname{curl}(-\Psi \hat{k})
$$

where $\hat{k}$ is perpendicular to the plane of motion and the Oseen linearization of the equations of motion can be written in terms of $\Psi$ as

$$
\nabla^{2}\left(\nabla^{2}+R \frac{\partial}{\partial x}\right) \Psi=0, \quad \nabla^{2} \equiv \frac{\partial^{2}}{\partial x^{2}}+\frac{\partial^{2}}{\partial y^{2}} .
$$

$R$ is the Reynolds number based on the free stream speed at infinity, the length scale for the boundary geometry under consideration, and the kinematic viscosity. The Oseen approximation is suitable for fluid domains of infinite extent in which the flow converges to a uniform stream at infinity, that is $\Psi \sim y$ as $x^{2}+y^{2} \rightarrow \infty$. The essence of the approximation is to account for the forced convection produced by the uniform stream, and the convection term $R(\mathbf{q} \cdot \nabla) \mathbf{q}$ which appears in the NavierStokes equation is linearized by replacement with $-R(\hat{\imath} \cdot \bar{\nabla}) \mathbf{q}$. Not only does the Oseen approximation resolve the Stokes paradox, but to leading order the solutions provide uniformly valid composite expressions for the stream function at small Reynolds numbers $R$. For example, the boundary value problem for flow past a circular cylinder may be expressed in terms of polar coordinates as

$\nabla^{2}\left(\nabla^{2}+R \frac{\partial}{\partial x}\right) \Psi=0, \quad \nabla^{2} \equiv \frac{\partial^{2}}{\partial r^{2}}+\frac{1}{r} \frac{\partial}{\partial r}+\frac{1}{r^{2}} \frac{\partial^{2}}{\partial \theta^{2}}, \quad \frac{\partial}{\partial x} \equiv \cos \theta \frac{\partial}{\partial r}-\frac{\sin \theta}{r} \frac{\partial}{\partial \theta}$. 
The boundary conditions are

$$
\Psi=\frac{\partial \Psi}{\partial r}=0, \quad \text { at } r=1, \quad \Psi \sim y=r \sin \theta, \quad \text { as } r \rightarrow \infty .
$$

An exact solution for $\Psi$ is not possible, but a uniformly valid approximation for small Reynolds numbers $R$ is provided by

$$
\begin{aligned}
\Psi=\sin \theta\{r- & \frac{1}{r} \frac{4}{R}+\left\{\log \left(\frac{4}{R}\right)-\gamma+\frac{1}{2}\right\}^{-1} \\
& \left.\times\left[e^{-(R r / 2) \cos \theta} K_{1}\left(\frac{R r}{2}\right)-\frac{1}{r}\left[\frac{2}{R}-\frac{R}{4}+\frac{1}{4} R\left[\gamma-\log \left(\frac{4}{R}\right)\right]\right]\right]\right\}
\end{aligned}
$$

where $K_{1}$ is the modified Bessel function of the second kind and first order, and $\gamma$ is Euler's constant. This expression $\Psi$ satisfies the Oseen equation, the outer boundary condition exactly, and satisfies the inner boundary conditions correct to $O(R \log R)$ as $R \rightarrow 0$.

Even though it is seldom required in practice there are few complete solutions of the Oseen equation in the sense that the boundary conditions are satisfied exactly. One such solution, which has a three-dimensional analogue [1] is the flow past a parabolic cylinder. The simplest method for deriving the stream function is to define parabolic coordinates by $x+i y=(\xi+i \eta)^{2}$ and seek a similarity solution for $\Psi$ in the form $\Psi=\eta f(\xi)$. The parabolic cylinder is typified by $\xi=\xi_{0}$, and the details of the solution are straightforward. The stream function $\Psi$ in $(\xi, \eta)$ coordinates is represented by

$$
\begin{aligned}
\Psi=2 \eta\{\xi & -\xi_{0}-\frac{1}{(\pi R)^{1 / 2} \operatorname{erfc}\left(\xi_{0} R^{1 / 2}\right)} \\
& \left.\times\left[e^{-R \xi^{2}}-\xi(\pi R)^{1 / 2} \operatorname{erfc}\left(\xi R^{1 / 2}\right)-e^{-R \xi_{0}^{2}}+\xi_{0}(\pi R)^{1 / 2} \operatorname{erfc}\left(\xi_{0} R^{1 / 2}\right)\right]\right\}
\end{aligned}
$$

where the complementary error function is defined by

$$
\operatorname{erfc} s=\frac{2}{\pi^{1 / 2}} \int_{s}^{\infty} e^{-u^{2}} d u
$$

The particular case $\xi_{0}=0$, corresponds to the degenerate situation of a semi-infinite flat plate $y=0, x<0$ and

$$
\Psi=2 \eta\left\{\xi-\frac{1}{(\pi R)^{1 / 2}}\left[e^{-R \xi^{2}}-1-\xi\left(\pi R^{1 / 2}\right) \operatorname{erfc}(\xi R)^{1 / 2}\right]\right\} .
$$

This solution has been derived in a different way in [2].

One major advantage of the Oseen equation is that since solutions are analytic in the fluid domain the two second-order operators in (B) commute which in turn implies a decomposition of the solution in the form

$$
\Psi=u+v
$$

where

$$
\nabla^{2} u=0, \quad\left(\nabla^{2}+R \frac{\partial}{\partial x}\right) v=0
$$


The latter equation can be reduced to the Helmholtz diffusion equation by the transformation

$$
\left(\nabla^{2}-\frac{R^{2}}{4}\right)\left(v e \frac{R x}{2}\right)=0 .
$$

This decomposition facilitates the construction of solutions of the partial differential equation but the term $e \frac{R x}{2}$ inevitably creates a spreading on constants contained in the solution in that the boundary conditions reduce to difference equations or sets of infinite equations for the undetermined coefficients.

The Burgers approximation. If $\Omega(z)=\alpha+i \beta$ is the complex potential for the streaming flow past a fixed boundary in the plane, the Burgers approximation seeks to replace the convection term by the forced convection produced by the potential flow, so the inner boundary conditions are partially satisfied to the extent vorticity is convected round the boundary and not through it, as in the Oseen flow. The linearized Burgers equation is given by (see $[3(b)])$

$$
R \frac{\partial\left(\beta, \nabla^{2} \Psi\right)}{\partial(x, y)}=\nabla^{4} \Psi
$$

which can be mapped into the plane of the velocity potential $\alpha$ and stream function $\beta$ by the equation

$$
w_{\alpha \alpha}+w_{\beta \beta}+R w_{\alpha}=0
$$

where the vorticity

$$
w=\nabla^{2} \Psi=\left(\Psi_{\alpha \alpha}+\Psi_{\beta \beta}\right) / \frac{\partial(x, y)}{\partial(\alpha, \beta)} .
$$

Complete exact solutions of these equations satisfying no slip boundary condition are the Oseen flow past a semi-infinite flat plate as defined by $(G)$, and stagnation point flow towards a plane wall. While it is not difficult to determine solutions for $w$ the analysis can become cumbersome if not intractable when calculating $\Psi$ from the Poisson equation (L) subject to the no slip boundary conditions. This is due to the presence of the scale factor $\frac{\partial(x, y)}{\partial(\alpha, \beta)}$ arising from the mapping transformation.

The present paper describes a Burgers type linearization for a complex variable form of the two-dimensional, steady flow Navier-Stokes equations which results in a conformally invariant form of the Oseen equation where the independent variables consist of the velocity potential and stream function for the corresponding irrotational flow. In terms of accuracy at small Reynolds numbers this method is essentially neither better or worse than conventional theories but has the advantage that the tractability of boundary value problems is substantially increased, and at the same time the method is applicable to a general variety of flow geometries which would be virtually intractable using existing approximations.

Complex variable formulation of the Navier-Stokes equations. The equations of motion for the steady flow of an incompressible viscous fluid can be written in the form

$$
\begin{array}{r}
-[\mathbf{q} \times \operatorname{curl} \mathbf{q}]=-\nabla B+\nu \nabla^{2} \mathbf{q} \\
\operatorname{div} \mathbf{q}=0, \quad B=\frac{p}{\rho_{0}}+\frac{1}{2}|\mathbf{q}|^{2},
\end{array}
$$


where $\mathbf{q}$ is the fluid velocity, $p$ the pressure, $\rho_{0}$ the density, $\nu$ the kinematic viscosity, and $B$ the Bernoulli function or total head of pressure. (In this way, part of the convection terms have been absorbed into the pressure gradient.) For two-dimensional flow a stream function $\Psi(x, y)$ can be introduced by

$$
\mathbf{q}=u \hat{\imath}+v \hat{\jmath}=\operatorname{curl}(-\Psi \hat{k})=-\Psi_{y} \hat{\imath}+\Psi_{x} \hat{\jmath} .
$$

The components of Eq. (1) are

$$
\begin{aligned}
& -\Psi_{x} \nabla^{2} \Psi=-B_{x}-\nu \frac{\partial}{\partial y} \nabla^{2} \Psi \\
& -\Psi_{y} \nabla^{2} \Psi=-B_{y}+\nu \frac{\partial}{\partial x} \nabla^{2} \Psi .
\end{aligned}
$$

If $z=x+i y, \bar{z}=x-i y$ then

$$
2 \frac{\partial}{\partial z} \equiv \frac{\partial}{\partial x}-i \frac{\partial}{\partial y}, \quad 2 \frac{\partial}{\partial \bar{z}} \equiv \frac{\partial}{\partial x}+i \frac{\partial}{\partial y}
$$

and Eq. (4), (5) can be combined in complex form to produce the single complex equation

$$
-4 \Psi_{\bar{z}} \Psi_{z \bar{z}}=-B_{\bar{z}}+4 \nu i \Psi_{z \overline{z z}}
$$

where $\nabla^{2} \Psi=\Psi_{x x}+\Psi_{y y}=4 \Psi_{z \bar{z}}$. In addition, from (3),

$$
u+i v=2 i \Psi_{\bar{z}}
$$

If a real function $\phi(x, y)$ is defined by $B=-\nu \nabla^{2} \phi=-4 \nu \phi_{z \bar{z}}$ then (7) can be written as

$$
\phi_{z \overline{z z}}+i \Psi_{z \overline{z z}}+\frac{1}{\nu} \Psi_{\bar{z}} \Psi_{z \bar{z}}=0
$$

which implies

$$
\phi_{\overline{z z}}+i \Psi_{\overline{z z}}+\frac{1}{2 \nu} \Psi_{\bar{z}}^{2}=f(\bar{z})
$$

where $f$ is an arbitrary function of $\bar{z}$ analytic in some domain of the fluid region. Equation (10) is the complex form of the two-dimensional Navier-Stokes equation and was first derived in a slightly modified form by Legendre [4]. Elimination of $\phi$ from (10) produces the usual vorticity equation for the stream function represented by

$$
\nu \nabla^{4} \Psi=\frac{\partial\left(\Psi, \nabla^{2} \Psi\right)}{\partial(x, y)}
$$

In fact if (10) is slightly modified to

$$
\phi_{\overline{z z}}+i \Psi_{\overline{z z}}+\frac{1}{2 \nu} \Psi \frac{2}{z}=z A(\bar{z})+B(\bar{z})
$$

where $A(\bar{z}), B(\bar{z})$ are arbitrary then elimination of $\phi$ from (12) produces (11) so that $\Psi$ is a solution of the vorticity equation. It is convenient to write

$$
\Psi=\chi+h(z)+\bar{h}(\bar{z})
$$


where $\chi$ is real, and $h(z)$ is analytic in the fluid region. $h(z)$ is related to the complex potential for irrotational flow past the particular boundary under consideration. Replacing $\Psi$ by $\chi$ in (12) gives the equation

$$
\phi_{\overline{z z}}+i \chi_{\overline{z z}}+\frac{1}{2 \nu} \chi_{\bar{z}}^{2}+\frac{1}{\nu} \bar{h}^{\prime}(\bar{z}) \chi_{\bar{z}}=z A(\bar{z})+C(\bar{z})
$$

where

$$
C(\bar{z})=B(\bar{z})-i \bar{h}^{\prime \prime}(\bar{z})-\frac{1}{2 \nu}\left[\bar{h}^{\prime}(\bar{z})\right]^{2} .
$$

It is also appropriate to introduce a real function $F=\phi+\frac{1}{4 \nu} \chi^{2}$, and since

$$
\frac{\partial^{2}}{\partial \bar{z}^{2}}\left(\frac{\chi^{2}}{4 \nu}\right)=\frac{\chi}{2 \nu} \chi \overline{z z}+\frac{\chi \frac{2}{z}}{2 \nu}
$$

Eq. (14) can be rewritten as

$$
F_{\overline{z z}}+\left(i-\frac{1}{2 \nu} \chi\right) \chi \overline{z \bar{z}}+\frac{1}{\nu} \bar{h}^{\prime}(\bar{z}) \chi_{\bar{z}}=z A(\bar{z})+C(\bar{z}) .
$$

At this point to make further progress Eq. (17) is linearized with respect to the function $\chi$ which appears in the second term. $\chi$ is replaced by $-2[h(z)+\bar{h}(\bar{z})]$ and the resulting equation is

$$
F_{\overline{z z}}+\left\{i+\frac{1}{\nu}[h(z)+\bar{h}(\bar{z})]\right\} \chi_{\overline{z z}}+\frac{1}{\nu} \bar{h}^{\prime}(\bar{z}) \chi_{\bar{z}}=z A(\bar{z})+C(\bar{z}) .
$$

This linearization amounts to a Burgers type approximation where the convecting stream function $\Psi$ is replaced by the forced convection of the corresponding potential flow which in this case is $-[h(z)+\bar{h}(\bar{z})]$. Equation (18) can be integrated to give

$$
F_{\bar{z}}+\left\{i+\frac{1}{\nu}[h(z)+\bar{h}(\bar{z})]\right\} \chi_{\bar{z}}=z A_{1}(\bar{z})+C_{1}(\bar{z})+K(z)
$$

where $A_{1}^{\prime}(\bar{z})=A(\bar{z}), C_{1}^{\prime}(\bar{z})=C(\bar{z})$, and $K(z)$ is analytic in the fluid region. Equation (19) can be simplified by writing

$$
\begin{aligned}
F & =\bar{z} K(z)+z \bar{K}(\bar{z})+G-\frac{h(z)}{\nu} \bar{h}_{1}(\bar{z})-\frac{h_{1}(z)}{\nu} \bar{h}(\bar{z}) \\
\chi & =H+h_{1}(z)+\bar{h}_{1}(\bar{z})
\end{aligned}
$$

where $G, H$ are real and $h_{1}(z)$ is analytic in the fluid region. If Eqs. (20), (21) are substituted in (19) the resulting equation is

$$
G_{z}+\left\{i+\frac{1}{\nu}[h(z)+\bar{h}(\bar{z})]\right\} H_{\bar{z}}=\frac{1}{\nu} h_{1}(z) \bar{h}^{\prime}(\bar{z})
$$

where the arbitrary functions $A_{1}(\bar{z}), C_{1}(\bar{z})$ have been chosen so that

$$
A_{1}(\bar{z})=\bar{K}^{\prime}(\bar{z}), \quad C_{1}(\bar{z})=\bar{h}(\bar{z}) \bar{h}_{1}^{\prime}(\bar{z}) .
$$

With $\zeta=\alpha+i \beta=h(z)$ Eq. (33) can be mapped into the $\zeta$-plane by the equation

$$
G_{\bar{\zeta}}+\left[i+\frac{1}{\nu}(\zeta+\bar{\zeta})\right] H_{\bar{\zeta}}=\frac{1}{\nu} h_{1}(z)=\frac{1}{\nu} H_{1}(\zeta)
$$


where $H_{1}(\zeta)$ is essentially arbitrary. Equation (24) differentiated with respect to $\zeta$ yields

$$
G_{\zeta \bar{\zeta}}+\left[i+\frac{1}{\nu}(\zeta+\bar{\zeta})\right] H_{\zeta \bar{\zeta}}+\frac{1}{\nu} H_{\bar{\zeta}}=\frac{1}{\nu} H_{1}^{\prime}(\zeta)
$$

and the complex conjugate equation is

$$
G_{\zeta \zeta}+\left[\frac{1}{\nu}(\zeta+\bar{\zeta})-i\right] H_{\zeta \zeta}+\frac{1}{\nu} H_{\zeta}=\frac{1}{\nu} \bar{H}_{1}^{\prime}(\bar{\zeta}),
$$

so that elimination of $G_{\zeta \zeta}$ from (25), (26) gives

$$
2 i H_{\zeta \zeta}+\frac{1}{\nu}\left(H_{\zeta}-H_{\zeta}\right)=\frac{1}{\nu}\left[H_{1}^{\prime}(\zeta)-\bar{H}_{1}^{\prime}(\bar{\zeta})\right] .
$$

Now

$$
2 \frac{\partial}{\partial \bar{\zeta}} \equiv \frac{\partial}{\partial \alpha}+i \frac{\partial}{\partial \beta}, \quad 2 \frac{\partial}{\partial \zeta} \equiv \frac{\partial}{\partial \alpha}-i \frac{\partial}{\partial \beta}
$$

and (27) can now be put in the form

$$
H_{\alpha \alpha}+H_{\beta \beta}+\frac{2}{\nu} H_{\beta}=2 M
$$

where $H_{1}^{\prime}(\zeta)-\bar{H}_{1}^{\prime}(\bar{\zeta})=2 M \nu i$ so that $M$ satisfies

$$
M_{\alpha \alpha}+M_{\beta \beta}=0 .
$$

Elimination of $M$ from (29), (30) produces the equation for $H$ in the form

$$
\left(\frac{\partial^{2}}{\partial \alpha^{2}}+\frac{\partial^{2}}{\partial \beta^{2}}\right)\left(\frac{\partial^{2}}{\partial \alpha^{2}}+\frac{\partial^{2}}{\partial \beta^{2}}+\frac{2}{\nu} \frac{\partial}{\partial \beta}\right) H=0 .
$$

Since the operators (31) commute, the solution for $H$ can be decomposed in the form

$$
H=u+v
$$

where $u, v$ are solutions of

$$
u_{\alpha \alpha}+u_{\beta \beta}=0, \quad v_{\alpha \alpha}+v_{\beta \beta}+\frac{2}{\nu} v_{\beta}=0
$$

or equivalently the two-dimensional Helmholtz diffusion equation

$$
\left(\frac{\partial^{2}}{\partial \alpha^{2}}+\frac{\partial^{2}}{\partial \beta^{2}}-\frac{1}{\nu^{2}}\right)\left(v e^{\beta / \nu}\right)=0 .
$$

Again from (13), (21), $\Psi$ is expressible as

$$
\Psi=H+h_{1}(z)+\bar{h}_{1}(\bar{z})+h(z)+\bar{h}(\bar{z}),
$$

and because $\Psi-H$ is a two-dimensional harmonic, $\Psi$ satisfies the same equation as $H$, viz.

$$
\left(\frac{\partial^{2}}{\partial \alpha^{2}}+\frac{\partial^{2}}{\partial \beta^{2}}\right)\left(\frac{\partial^{2}}{\partial \alpha^{2}}+\frac{\partial^{2}}{\partial \beta^{2}}+\frac{2}{\nu} \frac{\partial}{\partial \beta}\right) \Psi=0 .
$$

Hence the Burgers type approximation carried out in this section results in a conformally invariant form of the Oseen equation in which the stream function $\Psi$ in the governing equations has been replaced by $-2 \alpha$, the forced convection represented by 
the potential flow. In fact the complex potential for the irrotational flow is represented by

$$
w(z)=-2 i(\alpha+i \beta) .
$$

The preceding analysis has been carried out on the assumption $\frac{1}{\nu}>0$, and the limit $\nu \rightarrow \infty$ does not express conformal invariance of the biharmonic equation but merely reaffirms the fact that the two-dimensional Laplace equation is invariant under conformal mapping. It follows from Eq. (36) that known results for the Oseen equation can be mapped into the plane of the velocity potential and stream function for irrotational flow. It is noted that if $2 \alpha=-y, 2 \beta=x$, then Eq. (36) reduces to the Oseen equation for the stream function $\Psi$ in which the forced convecting stream function is $-2 \alpha=y$. Some examples of specific flows arising from Eq. (36) are outlined in the next section.

Boundary value problems. The nondimensional form of (36) is expressed by

$$
\left(\frac{\partial^{2}}{\partial \alpha^{2}}+\frac{\partial^{2}}{\partial \beta^{2}}\right)\left(\frac{\partial^{2}}{\partial \alpha^{2}}+\frac{\partial^{2}}{\partial \beta^{2}}+R \frac{\partial}{\partial \beta}\right) \Psi=0,
$$

where $R$ is the Reynolds number based on the parameters contained in the forcing flow, geometry of the boundaries and the kinematic viscosity $\nu$. It is important that $R>0$, but there are solutions of (38) which depend only on $\alpha$ and are independent of $R$. These flows correspond to a generalization of plane Poiseuille flow between parallel planes under a constant gradient, to a channel flow between curved boundaries with constant total flux rate $Q$. For example if the channel is described by the coordinates $\alpha_{1} \leq \alpha \leq \alpha_{2},-\infty<\beta<\infty$, the boundary value problem is

$$
\left.\Psi\right|_{\alpha=\alpha_{1}}-\left.\Psi\right|_{\alpha=\alpha_{2}}=Q, \quad \frac{d \Psi}{d \alpha}=0, \quad \text { at } \alpha=\alpha_{1} \text { and } \alpha=\alpha_{2},
$$

and (38) reduces to

$$
\frac{d^{4} \Psi}{d \alpha^{4}}=0
$$

The solution for $\Psi$ is given by

$$
\Psi=\frac{Q \alpha}{\left(\alpha_{2}-\alpha_{1}\right)^{3}}\left\{\alpha_{1} \alpha_{2}-3 \alpha\left(\alpha_{1}+\alpha_{2}\right)+2 \alpha^{2}\right\} .
$$

To be more specific the mapping

$$
x+i y=(\alpha+i \beta)^{2},
$$

or equivalently

$$
x=\alpha^{2}-\beta^{2}, \quad y=2 \alpha \beta,
$$

transforms $\alpha=\alpha_{1}$ into the parabola

$$
x=\alpha_{1}^{2}-\frac{y^{2}}{4 \alpha_{1}^{2}},
$$

and similarly $\alpha=\alpha_{2}$ transforms into the parabola

$$
x=\alpha_{2}^{2}-\frac{y^{2}}{4 \alpha_{2}^{2}} .
$$


The stream function represented by (41) describes the flow in a curved channel bounded by confocal parabolas. It is also noted that this solution could not be predicted by conventional low Reynolds theory as there is no solution of the Stokes equations or Burgers equation in which the stream function coincides with one family of coordinate curves satisfying the conditions of no slip.

A rotlet interior to a corrugated cylinder. For flow in the presence of a corrugated boundary the transformation [5]

$$
z=x+i y=w+\varepsilon w^{n+1}, \quad w=\rho e^{i \phi}, \quad 0<m=\varepsilon(n+1)<1
$$

maps the interior of the unit circle $\rho \leq 1$ into the interior of a closed corrugated boundary with $n$ peaks ( $n$ is a positive integer) lying between the circles $r=1 \pm \varepsilon$. If the flow is stirred by a rotlet at the origin there is a complete Stokes solution which is described by the boundary value problem

$$
\begin{gathered}
\nabla^{4} \Psi=0, \quad \nabla^{2} \equiv \frac{1}{\left[1+m^{2} \rho^{2 n}+2 m \rho^{n} \cos n \phi\right]}\left[\frac{\partial^{2}}{\partial \rho^{2}}+\frac{1}{\rho} \frac{\partial}{\partial \rho}+\frac{1}{\rho^{2}} \frac{\partial^{2}}{\partial \phi^{2}}\right] \\
\psi \sim \log \rho, \quad \text { as } \rho \rightarrow 0, \quad \Psi=\text { constant }, \quad \frac{\partial \Psi}{\partial \rho}=0 \quad \text { at } \rho=1 .
\end{gathered}
$$

The solution for the stream function is readily found to be

$$
\Psi=\log \rho-\frac{\left[\rho^{2}-\varepsilon^{2} \rho^{2 n+2}\right]}{2\left[1-(n+1) \varepsilon^{2}\right]}
$$

so that the fluted columns, $\rho=$ constant, coincide with the streamlines.

Now with $\alpha=\log \rho, \beta=\phi$, Eq. (38) takes the form

$$
\left[\rho \frac{\partial}{\partial \rho}\left(\rho \frac{\partial}{\partial \rho}\right)+\frac{\partial^{2}}{\partial \phi^{2}}\right]\left[\rho \frac{\partial}{\partial \rho}\left(\rho \frac{\partial}{\partial \rho}\right)+\frac{\partial^{2}}{\partial \phi^{2}}+R \frac{\partial}{\partial \phi}\right] \Psi=0
$$

and the boundary conditions are

$$
\Psi \sim \log \rho, \quad \text { as } \rho \rightarrow 0, \quad \Psi=\text { constant }, \quad \frac{\partial \Psi}{\partial \rho}=0 \quad \text { at } \rho=1 .
$$

The solution for $\Psi$ is given by

$$
\Psi=\log \rho-\frac{1}{2} \rho^{2}
$$

so the streamlines coincide with $\rho=$ constant. The two solutions for $\Psi$ represented by (49) and (52) differ by the term

$$
\frac{\varepsilon^{2}\left[\rho^{2 n+2}-(n+1) \rho^{2}\right]}{2\left[1-(n+1) \varepsilon^{2}\right]}
$$

which is uniformly small if $0<\varepsilon \ll 1$, or $n$ is large. The two solutions are identical in the case $\varepsilon=0$, and the outer boundary is a circle, which in turn is an exact solution of the Navier-Stokes equations. 
Flow past a parabolic cylinder. The stream function for flow of a parabolic cylinder

$$
x=\xi_{0}^{2}-\frac{y^{2}}{4 \xi_{0}^{2}}
$$

is expressed by

$$
\alpha=2 \eta(\xi-\xi), \quad x+i y=(\xi+i \eta)^{2} .
$$

The corresponding form of $\beta$ is

$$
\beta=-\xi^{2}+\eta^{2}+2 \xi \xi_{0}
$$

It follows the solution for the linearized equation (38) subject to no slip boundary conditions on $\xi=\xi_{0}$ with a uniform stream at infinity is expressed by $(G)$ replacing $\xi$ with $\left(\xi-\xi_{0}\right)$; that is

$$
\Psi=2 \eta\left\{\xi-\xi_{0}-\frac{1}{(\pi R)^{1 / 2}}\left[e^{-R\left(\xi-\xi_{0}\right)^{2}}-1-\left(\xi-\xi_{0}\right)(\pi R)^{1 / 2} \operatorname{erfc}\left[\left(\xi-\xi_{0}\right) R^{1 / 2}\right]\right]\right\} .
$$

Flow between two nonconcentric circular cylinders. In the notation of Jeffery [3(a), 6] the appropriate system of bipolar coordinates for flow between nonconcentric circular cylinders is defined by

$$
\alpha+i \beta=\log \left\{\frac{x+i(y+b)}{x+i(y-b)}\right\}
$$

where

$$
x=\frac{b \sin \beta}{\cosh \alpha-\cos \beta}, \quad y=\frac{b \sinh \alpha}{\cosh \alpha-\cos \beta} .
$$

The inner cylinder is typified by $\alpha=\alpha_{1}$ and the outer by $\alpha=\alpha_{2}$. If the radii are $r_{1}$, $r_{2}$ and the distances of the centres from the origin $d_{1}, d_{2}$, then

$$
\left.\begin{array}{ll}
r_{1}=b \operatorname{cosech} \alpha_{1}, & r_{2}=b \operatorname{cosech} \alpha_{2} \\
d_{1}=b \operatorname{coth} \alpha_{1}, & d_{2}=b \operatorname{coth} \alpha_{2} .
\end{array}\right\}
$$

If the angular velocities of the cylinders are $w_{1}, w_{2}$ the appropriate boundary conditions are

$$
\begin{array}{llll}
\psi=\psi_{1} & \text { a constant }, & \frac{\partial \psi}{\partial \alpha}=\frac{-b w_{1} \operatorname{cosech} \alpha_{1}}{\cosh \alpha_{1}-\cos \beta} & \text { at } \alpha=\alpha_{1} \\
\psi=\psi_{2} & \text { a constant }, & \frac{\partial \psi}{\partial \alpha}=\frac{-b w_{2} \operatorname{cosech} \alpha_{2}}{\cosh \alpha_{2}-\cos \beta} & \text { at } \alpha=\alpha_{2} .
\end{array}
$$

A suitable solution of (38) is represented by

$$
\psi=\operatorname{Re} \sum_{n=0}^{\infty} F_{n}(\alpha) e^{i n \beta}
$$

where the complex function $F_{n}(\alpha)$ satisfies

$$
\left(\frac{d^{2}}{d \alpha^{2}}-n^{2}\right)\left(\frac{d^{2}}{d \alpha^{2}}-n^{2}+i R n\right) F_{n}(\alpha)=0
$$


for $n=0,1,2, \ldots$. The solutions are

$$
F_{n}(\alpha)=A_{n} e^{n \alpha}+B_{n} e^{-n \alpha}+C_{n} e^{\lambda_{n} \alpha}+D_{n} e^{-\lambda_{n} \alpha}
$$

where $n \geq 1$, the coefficients $A_{n}, B_{n}, C_{n}, D_{n}$ are complex constants and $\lambda_{n}^{2}=n^{2}-i R n$. For $n=0$

$$
F_{0}(\alpha)=A_{0}+B_{0} \alpha+C_{0} \alpha^{2}+D_{0} \alpha^{3} .
$$

Utilizing the boundary conditions the constants $A_{n}, B_{n}, C_{n}, D_{n}$ are determined by straightforward Fourier analysis. Finally it is noted that if $\alpha_{1}, \alpha_{2}$ are both positive the cylinders are internally separated while if $\alpha_{1}>0, \alpha_{2}<0$, the cylinders are separated externally.

Flow past a fixed cylinder with finite cross section due to a uniform stream at infinity. From Eq. (E), the stream function $\psi$ defined by

$$
\begin{aligned}
\psi=\sin \phi\{\rho & -\frac{1}{\rho}+\frac{4}{R\left[\log \left(\frac{4}{R}\right)-\gamma+\frac{1}{2}\right]} \\
& \left.\times\left[e^{-(R \rho / 2) \cos \phi} K_{1}\left(\frac{R \rho}{2}\right)-\frac{1}{\rho}\left[\frac{2}{R}-\frac{R}{4}+\frac{1}{4} R\left[\gamma-\log \left(\frac{4}{R}\right)\right]\right]\right]\right\}
\end{aligned}
$$

where $\beta+i \alpha=\rho e^{i \phi}=f(\bar{z})$, where

$$
\alpha=\frac{f(\bar{z})-\bar{f}(z)}{2 i} \sim y \quad \text { as }|z| \rightarrow \infty
$$

defines a composite uniformly valid approximation to the flow past the cylinder $\rho=1$, in which the inner boundary conditions on $\rho=1$ are satisfied to $O(R \log R)$ as $R \rightarrow 0$. The flow at infinity converges to a uniform stream. A possible form for $\bar{f}(z)$ is expressed by

$$
\bar{f}(z)=-z+\sum_{n=0}^{\infty} A_{n} z^{-n}
$$

where $\bar{f}^{\prime}(z) \neq 0, \infty$, in the fluid region.

Conclusion. The preceding examples support the claim that the linearization of the Navier-Stokes equations are given by Eq. (38), leads to a simplification of the analytical process in calculating solutions for the stream function at small Reynolds numbers. The approximation is also applicable to a wide range of flow geometries which would be prohibitive in terms of mathematical tractability by both conventional Oseen and Burgers approximations.

\section{REFERENCES}

[1] J. Wilkinson, $A$ note on the Oseen approximation for a paraboloid in a uniform stream parallel to its axis, Quart. J. Mech. Appl. Math. 8, 415-421 (1955)

[2] J. A. Lewis and G. F. Carrier, Some remarks on the flat plate boundary layer Quart. Appl. Math. 7 228-234, (1949)

[3] H. L. Dryden, F. P. Murnaghan, and H. Bateman, Hydrodynamics, Dover, New York, 1956, (a) pp. 233-235, (b) pp. 265-266 
[4] R. Legendre, Solution plus complète du problème Blasius, Comptes Rendus 228, June 1949, 2008-2010

[5] D. E. R. Godfrey, Theoretical Elasticity and Plasticity for Engineers, Thames and Hudson, London, 1959 , pp. 58-59

[6] G. B. Jeffery, The rotation of two circular cylinders in a viscous fluid, Proc. Roy. Soc. London A101, 169-174 (1922) 